\title{
Cenário para treinamento por simulação sobre comunicação de notícias difíceis: um estudo de validação
}

\author{
Scenario for simulation training on the communication of hard news: A validation study \\ Escenario para el entrenamiento de simulación sobre la comunicación de noticias difíciles: estudio de \\ validación
}

\author{
Daiane de Assis Flausino ${ }^{1}$ (1) \\ Andressa Rueda de Oliveira ${ }^{1}$ (B) \\ Maira Deguer Misko²
} Aline Helena Appoloni Eduardo ${ }^{3}$ (])

1. Universidade Federal de São Carlos, Departamento de Enfermagem. São Carlos, SP, Brasil.

2. Universidade Estadual de Campinas, Faculdade de Enfermagem. Campinas, SP, Brasil.

3. Universidade Federal de São Carlos, Programa de Pós-Graduação em Enfermagem. São Carlos, SP, Brasil.
Autor correspondente:

Aline Helena Appoloni Eduardo.

E-mail: alinehaeduardo@ufscar.br

Recebido em 08/03/2021.

Aprovado em 16/09/2021.

\section{Resumo}

Objetivo: construir e validar um cenário de simulação realística sobre comunicação de notícias difíceis no contexto de cuidados paliativos para graduandos de enfermagem. Método: estudo metodológico, que seguiu três etapas; construção do cenário, fundamentada nos referenciais sobre simulação, comunicação de notícias difíceis e cuidados paliativos; validação de conteúdo, na qual nove juízes analisaram a adequação do cenário, por meio de uma escala Likert, para computar o índice Validade de Conteúdo (IVC) e, de sugestões descritas no instrumento de análise do cenário; teste do cenário, que ocorreu com 15 estudantes de graduação em enfermagem que o analisaram pela Escala de Design da Simulação (EDS). Nesta amostra, a análise da fidedignidade da escala foi verificada pelo alfa de Cronbach. Resultados: os juízes consideraram o conteúdo do cenário adequado. O IVC do cenário foi 0,84. Após o teste do cenário, os estudantes apontaram a adequação do design da simulação considerando-a adequada para a resolução do problema. $O$ alfa de Cronbach foi 0,89 para o total da EDS. Conclusão e implicações para a prática: o processo de construção e validação do cenário sobre comunicação de notícias difíceis resultou na produção de um material válido e consistente para ensino e pesquisas na área de cuidados paliativos.

Palavras-chave: Treinamento por Simulação; Comunicação; Enfermagem; Estudos de Validação; Cuidados Paliativos.

\section{Abstract}

Objective: to build and validate a realistic simulation scenario about the communication of hard news in the context of palliative care for undergraduate Nursing students. Method: a methodological study that followed three stages; construction of the scenario, which was based on the frameworks about simulation, communication of hard news and palliative care; content validation, in which nine judges analyzed adequacy of the scenario, through a Likert scale, to calculate the Content Validity Index (CVI) and of the suggestions described in the scenario analysis instrument; and scenario test, which was conducted with 15 undergraduate Nursing students who analyzed it using the Simulation Design Scale (SDS). In this sample, the analysis of the scale's reliability was verified by Cronbach's Alpha. Results: the judges considered the scenario's content as adequate. The scenario's CVI was 0.84. After testing the scenario, the students pointed out the simulation design's suitability and considered it adequate to solve the problem. The overall Cronbach's alpha for the SDS scale was 0.89 . Conclusion and implications for the practice: the process of constructing and validating the scenario on the communication of hard news resulted in the production of valid and consistent material for teaching and research in the area of Palliative Care.

Keywords: Simulation Training; Communication; Nursing; Validation Studies; Palliative Care.

\section{Resumen}

Objetivo: construir y validar un escenario de simulación realista sobre la comunicación de noticias difíciles en el contexto de los cuidados paliativos para estudiantes de enfermería. Método: estudio metodológico, que se desarrolló en tres etapas; construcción del escenario, basada en referencias sobre simulación, comunicación de noticias difíciles y cuidados paliativos; validación de contenido, en la que nueve jueces analizaron la adecuación del escenario, a través de una escala Likert, para computar el Índice de Validez de Contenido (IVC) y de las sugerencias descriptas en el instrumento de análisis de escenarios; prueba de escenario, que se llevó a cabo con 15 estudiantes de licenciatura en enfermería que lo analizaron mediante la Escala de Diseño de Simulación (EDS). En esta muestra, el análisis de confiabilidad de la escala se verificó por el alfa de Cronbach. Resultados: los jueces consideraron adecuado el contenido del escenario. El CVI del escenario fue 0,84. Luego de testear el escenario, los estudiantes señalaron la idoneidad del diseño de la simulación y lo consideraron adecuado para resolver el problema. El alfa de Cronbach fue de 0,89 para la EDS total. Conclusión e implicaciones para la práctica: el proceso de construcción y validación del escenario sobre la comunicación de noticias difíciles resultó en la producción de material válido y consistente para la docencia e investigación en el área de cuidados paliativos.

Palabras-clave: Entrenamiento Simulado; Comunicación; Enfermería; Estudio de Validación; Cuidados Paliativos. 


\section{INTRODUÇÃO}

O desenvolvimento das relações humanas está intimamente relacionado à capacidade de comunicação. Cotidianamente, os indivíduos adaptam suas expressões verbais e não verbais para se comunicar em diferentes contextos. Ainda assim, este processo pode sofrer interferências quando se trata da necessidade de comunicação com pessoas que estão recebendo cuidados de saúde e seus familiares ${ }^{1}$.

Deste modo, a equipe de saúde deve estar preparada para comunicar-se efetivamente nos diversos serviços que compõem a rede de atenção à saúde. Sendo assim, é evidente que a habilidade de comunicação é um atributo fundamental para a formação de profissionais de enfermagem, pois este profissional é agente ativo na comunicação de informações às pessoas e suas famílias que estão sob seus cuidados².

Além disso, os enfermeiros lidam com notícias difíceis e diversos momentos do contexto profissional, que podem ser antes, durante ou após as pessoas serem informadas de um diagnóstico, necessidade de tratamento ou resultado de exames, no manejo de consequências da condição de saúde imposta pela doença, no manejo de emoções geradas pela notícia difícil e durante os cuidados de final de vida ${ }^{3}$.

Uma notícia difícil é entendida como "qualquer informação que provavelmente altera drasticamente a perspectiva de futuro do paciente"4:1597, que contribui para a expressão de sentimentos de formas distintas e possui diferentes significados para quem as recebe ${ }^{3-5}$. Estas características tornam o processo de comunicação de notícias difíceis desafiador para os profissionais, bem como para o treinamento das habilidades comunicacionais durante a formação.

Em cuidados paliativos, a comunicação de notícias difíceis ocorre frequentemente para comunicação a respeito dos cuidados propostos, experiências impostas pelo tratamento, evolução e progressão da doença, questões próprias ao final de vida e promoção do conforto espiritual ${ }^{4,5}$. O alcance de bons resultados em cuidados paliativos depende da efetividade da comunicação entre equipe, usuários dos serviços de saúde e seus familiares.

Para realizar a comunicação nestes temas, é exigido dos profissionais habilidades de comunicação de notícias difíceis, tais habilidades requerem preparação e conhecimento de estratégias para fornecer informações angustiantes e manejar as reações resultantes destas informações ${ }^{6,7}$.

No entanto, ainda é desafiador afirmar que os profissionais de enfermagem e estudantes da área da saúde em geral, estão suficientemente preparados para se comunicar nestas situações ${ }^{8}$, uma vez que, durante a sua formação, não são oportunizadas vivências de comunicação neste contexto e, muitas vezes, esta habilidade é desenvolvida durante os anos da vida profissional, o que não é ideal, pois expõem os profissionais a momentos de sofrimento e estresse ${ }^{7}$. Conhecendo essa realidade, a inserção de metodologias ativas e inovadoras, como a simulação, no contexto formativo destes profissionais é crucial ${ }^{9}$.

Estudos que analisaram o efeito da simulação no preparo de profissionais para a comunicação de notícias difíceis em cuidados paliativos mostraram que habilidades fundamentais foram aprimoradas como desenvolvimento da escuta ativa, empatia e comunicação não verbal, além de melhorar a confiança para se comunicar e cuidar das pessoas em cuidados paliativos ${ }^{1,7}$.

Atualmente, o treinamento por simulação têm sido apontado por diversos estudos como a principal forma para ensino de profissionais da saúde para comunicação de notícias difíceis em cuidados paliativos ${ }^{10}$. Este tipo de experiência de aprendizagem oferece oportunidade para melhorar as habilidades comunicacionais de estudantes e profissionais, pois são atividades que representam situações reais ou potenciais da prática e permitem que os participantes desenvolvam ou aprimorem seus conhecimentos, habilidades e atitudes em um ambiente simulado ${ }^{11}$.

Apesar da essencialidade da prática, simular requer um planejamento adequado. Um dos elementos fundamentais para este planejamento é o cenário que precisa ser cuidadosamente desenvolvido para garantir a qualidade das experiências de aprendizagem $^{8,12}$.

Os cenários consistem na documentação dos detalhes que acontecerão durante a simulação, com informações que versam sobre a determinação de quem serão os participantes da simulação, instruções para estes participantes, objetivos de aprendizagem, preparação do ambiente, equipamentos, ferramentas para avaliação, caso clínico, duração entre outras. A detalhada construção destes cenários, acrescida da validação por especialistas é uma forma de elaborar cenários completos, passíveis de serem reproduzidos por diferentes grupos, contextos e capazes de representarem a realidade, resultando no sucesso da aprendizagem pela simulação.

Em vista disso, este estudo teve como objetivo construir e validar um cenário de simulação realística sobre comunicação de notícias difíceis no contexto de cuidados paliativos para graduandos de enfermagem.

\section{MÉTODO}

Trata-se de um estudo metodológico com vistas à construção e validação de cenário para treinamento por simulação, desenvolvido em três etapas: construção do cenário, validação de conteúdo do cenário por um painel de juízes e teste do cenário com graduandos de enfermagem. Foi desenvolvido no período de agosto de 2017 a dezembro de 2018.

Inicialmente, a construção do cenário foi apoiada nas evidências científicas sobre comunicação de notícias difíceis, a partir de uma revisão da literatura nas bases de dados para reunir os principais protocolos e referências sobre comunicação de notícias difíceis, que foram incorporadas na redação dos elementos fundamentais do cenário. O cenário elaborado considerou as recomendações da International Nursing Association for Clinical Simulation and Learning (INACSL), que propõe boas práticas e elementos fundamentais para a construção de cenários ${ }^{13}$. Além disso, a redação do cenário contemplou os elementos propostos para descrição de cenários, tal como descritos no Quadro $1^{14}$.

Para a validação de conteúdo, foi composto um painel de juízes a partir dos critérios de inclusão: ser profissional da saúde; 
Quadro 1. Definições dos elementos utilizados na descrição do cenário.

\begin{tabular}{|c|c|}
\hline $\begin{array}{l}\text { Elemento do } \\
\text { cenário }\end{array}$ & Definição \\
\hline Contexto & $\begin{array}{l}\text { Descrição do nome do cenário, perfil e pré-requisitos do participante, local onde a simulação será } \\
\text { realizada, propósito da simulação (educacional ou avaliação) e métodos empregados para avaliação do } \\
\text { participante. }\end{array}$ \\
\hline Responsáveis & Descrição dos facilitadores responsáveis pelo cenário. \\
\hline $\begin{array}{l}\text { Objetivos de } \\
\text { aprendizagem }\end{array}$ & $\begin{array}{l}\text { Descrição do que se pretende alcançar no âmbito da aprendizagem, descritos pelos objetivos geral } \\
\text { (amplo e reflete a proposta da simulação) e específico (relacionado às medidas de desempenho dos } \\
\text { participantes e compreendem habilidades técnicas e não técnicas). }\end{array}$ \\
\hline Duração & Definição de tempo para a realização da simulação e debriefing. \\
\hline $\begin{array}{l}\text { Preparação dos } \\
\text { participantes }\end{array}$ & $\begin{array}{l}\text { Descrição dos recursos empregados para a preparação dos participantes para a realização da simulação } \\
\text { (Exemplo: leituras recomendadas, participação em cursos, sessões didáticas, revisão de habilidades em } \\
\text { laboratório). }\end{array}$ \\
\hline $\begin{array}{l}\text { Modalidade de } \\
\text { simulação }\end{array}$ & $\begin{array}{l}\text { Discriminação quanto à modalidade de simulação: com uso de simuladores, atores, digital, em } \\
\text { laboratório, in situ ou híbrida. }\end{array}$ \\
\hline Design & $\begin{array}{l}\text { Descrição dos equipamentos e materiais necessários, detalhamento dos papeis dos participantes e } \\
\text { características dos simuladores, atores ou ambos. }\end{array}$ \\
\hline Pré-briefing & $\begin{array}{l}\text { Descrição das orientações gerais para a simulação, com a definição dos papeis entre os participantes, } \\
\text { apresentação do ambiente, equipamentos e documentações empregadas da simulação. }\end{array}$ \\
\hline Briefing & $\begin{array}{l}\text { Descrição das informações oferecidas imediatamente antes de iniciar a simulação com uma breve } \\
\text { informação sobre o caso a ser vivenciado. }\end{array}$ \\
\hline $\begin{array}{l}\text { Instruções para } \\
\text { a atriz }\end{array}$ & $\begin{array}{l}\text { Detalhamento das informações para atores sobre as características da personagem, falas, equipamentos } \\
\text { e caso. }\end{array}$ \\
\hline $\begin{array}{l}\text { Progressão da } \\
\text { simulação }\end{array}$ & $\begin{array}{l}\text { Descrição das cenas com as respectivas informações de tempo, falas do facilitador e atores, ações } \\
\text { esperadas dos participantes, planejamento para que os participantes sejam capazes de atingir os } \\
\text { objetivos (pistas), estratégias que auxiliem os participantes a manter o foco da simulação, se necessário, } \\
\text { e desfechos para finalização da simulação. }\end{array}$ \\
\hline Debriefing & Descrição do modelo de debriefing escolhido para a simulação. \\
\hline
\end{tabular}

possuir experiência clínica na área de cuidados paliativos, com atuação profissional na área por tempo mínimo de seis meses; ou ser pesquisador na área de comunicação e/ou cuidados paliativos, com publicações científicas sobre o(s) tema(s); ou ser facilitador em simulação clínica, com certificação desta formação ou ter experiência em facilitar atividades baseadas em simulação por tempo mínimo de seis meses. Não se estabeleceu critério de exclusão. O número estipulado para compor o painel de juízes foi entre seis e vinte, seguindo recomendações da literatura para estudos dessa natureza ${ }^{15}$.

Os juízes foram selecionados a partir da busca em publicações brasileiras sobre o tema, na Plataforma Lattes do Conselho Nacional de Desenvolvimento Científico e Tecnológico (http://lattes. cnpq.br) e pela estratégia bola de neve (quando um participante indica um potencial juiz a partir de seu conhecimento). Deste modo, nove juízes compuseram esta etapa.

Para o teste do cenário os participantes foram selecionados a partir dos critérios de inclusão: ser estudante de enfermagem; ter cursado ou estar cursando disciplinas nas áreas de comunicação e saúde da criança e possuir idade igual ou superior a 18 anos. O cenário considerou que o público alvo de cenário seria de estudantes que já tivessem tido algum nível de aproximação com a atenção à saúde da criança, devido às características do caso clínico elaborado, deste modo, foi intencionado pelas pesquisadoras que o teste ocorresse com participantes que atendessem estas características. Como critério de exclusão, estabeleceu-se: possuir alguma condição emocional ou psicológica que limitasse sua participação na simulação, este critério era avaliado no momento em que as pesquisadoras explicavam sobre os procedimentos do estudo e os participantes eram encorajados a verbalizar tais condições às pesquisadoras em qualquer momento.

Os estudantes foram selecionados por conveniência, a partir do recrutamento em redes sociais e inscrição voluntária. Por não haver padronização de número mínimo de participantes para estudos de teste de cenário, foi estabelecido pelas autoras um número mínimo de 10, embasadas em uma publicação sobre 
validação de cenário ${ }^{16}$. Portanto, compuseram esta etapa 15 estudantes.

A validação de conteúdo pelo painel de juízes ocorreu de forma virtual, estes foram contatados via e-mail e convidados a participar do estudo. Depois da aceitação, os instrumentos de coleta de dados foram enviados por um link de acesso de um formulário desenvolvido no aplicativo Google Forms ${ }^{\circledR}$. Neste formulário, além do cenário para ser analisado havia questões para caracterização dos juízes e questões para a validação de conteúdo do cenário.

As informações coletadas para caracterização dos juízes foram: formação profissional, experiência com simulação, cuidados paliativos e comunicação e tempo de experiência com cada tema. Para a validação de conteúdo do cenário, cada elemento foi analisado quanto à adequação, para isso, foram construídos critérios que permitiram essa análise. Cada um dos critérios, apresentados em sua totalidade na Tabela 1, era respondido segundo a escala 1 =totalmente adequado, $2=$ parcialmente adequado e 3=inadequado. Caso fosse de desejo dos juízes, era possível realizar anotações e sugestões quanto ao conteúdo, em espaços que foram deixados ao final da avaliação do conteúdo de cada elemento do cenário.

O teste do cenário ocorreu em uma unidade de simulação de uma universidade pública de ensino superior no interior do estado de São Paulo, entre participantes de uma estratégia educacional denominada "Oficina de Comunicação de Notícias Difíceis", na qual os mesmos assistiam a uma aula expositiva e dialogada sobre estratégias de comunicação de notícias difíceis e em seguida participavam da simulação. Cada oficina foi ofertada para cinco estudantes por vez e durante a simulação dois deles participavam voluntariamente da simulação ativamente e os demais assistiam. Antes de iniciar a Oficina, os estudantes eram informados sobre o estudo e, quando consentiam a participação, preenchiam um instrumento de caracterização e o TCLE. Ao final da simulação, os estudantes responderam a Escala de Design da Simulação (EDS) $)^{17}$.

O instrumento de caracterização dos estudantes contemplava informações sobre idade, período em que estavam no curso de Enfermagem e experiência prévia em atividades curriculares e/ou extracurriculares sobre comunicação de notícias difíceis.

Tabela 1. Índice de validade de conteúdo (IVC) do cenário de simulação de notícias difíceis. São Carlos, SP, Brasil, 2018. (n=09).

\begin{tabular}{|c|c|c|c|c|}
\hline \multirow{2}{*}{ Critérios de avaliação } & \multicolumn{3}{|c|}{ Adequação } & \multirow{2}{*}{ IVC } \\
\hline & 1 & 2 & 3 & \\
\hline As informações no contexto permitem a visualização geral do cenário & 7 & 2 & 0 & 0,78 \\
\hline Os objetivos de aprendizagem estão adequadamente descritos & 6 & 3 & 0 & 0,67 \\
\hline Os dados sobre o design do cenário estão completos & 5 & 4 & 0 & 0,56 \\
\hline As estratégias para preparação dos participantes são adequadas & 6 & 3 & 0 & 0,67 \\
\hline As informações oferecidas no pré-briefing e briefing são suficientes & 7 & 2 & 0 & 0,78 \\
\hline As instruções oferecidas para a atriz são suficientes & 4 & 7 & 0 & 0,44 \\
\hline As cenas estão condizentes com as falas e pistas & 9 & 0 & 0 & 1 \\
\hline As cenas permitem que os participantes realizem as ações esperadas & 9 & 0 & 0 & 1 \\
\hline Os desfechos estão claramente descritos & 9 & 0 & 0 & 1 \\
\hline A estrutura do debriefing está apropriada & 7 & 2 & 0 & 0,78 \\
\hline Coerência entre o caso clínico e objetivo da simulação & 9 & 0 & 0 & 1 \\
\hline Realismo do cenário & 7 & 2 & 0 & 0,78 \\
\hline Complexidade em relação ao nível de conhecimento e habilidades do aluno & 9 & 0 & 0 & 1 \\
\hline Informações fornecidas aos participantes antes da simulação & 7 & 2 & 0 & 0,78 \\
\hline Informações fornecidas aos participantes durante a simulação & 9 & 0 & 0 & 1 \\
\hline Apoio fornecido aos participantes durante a simulação & 8 & 1 & 0 & 0,89 \\
\hline Promoção do pensamento crítico & 9 & 0 & 0 & 1 \\
\hline Promoção da capacidade em empregar estratégias de comunicação & 8 & 1 & 0 & 0,89 \\
\hline Promoção da resolução autônoma de problemas & 9 & 0 & 0 & 1 \\
\hline IVC Total & & & & \\
\hline
\end{tabular}

*IVC: Índice de Validade de Conteúdo.

Fonte: dados de pesquisa. 
A EDS tem a finalidade de avaliar a estrutura dos cenários a partir da participação da simulação. Foi desenvolvida pela National League for Nursing, originalmente em inglês, mas foi traduzida, adaptada e validada para uso no Brasil. Trata-se de uma escala com 20 itens, composta por cinco fatores que avaliam: objetivos e informações, apoio, resolução de problemas, feedback e reflexão e realismo. Cada item é respondido por uma escala Likert, de cinco pontos que varia de $1=$ Discordo totalmente da afirmação a 5= Concordo totalmente com a afirmação, com possibilidade de resposta "não aplicável", quando a declaração expressa no item não diz respeito à simulação realizada. Para obtenção do escore, computa-se a média de cada fator e do total da escala, os resultados mais elevados representam melhor percepção do respondente em relação ao design do cenário ${ }^{17}$.

Os dados obtidos neste estudo foram compilados em uma planilha do programa Microsoft Excel ${ }^{\circledR}$ e analisados conforme especificidade da etapa da pesquisa (validação de conteúdo e teste do cenário).

Os dados resultantes da validação de conteúdo foram analisados de duas formas: análise das sugestões pelas pesquisadoras e computado o Índice de Validação de Conteúdo (IVC). A análise das sugestões consistiu na discussão destas entre as pesquisadoras para decisão da incorporação destas sugestões ao cenário. $O$ IVC foi verificado por cada critério de análise do cenário e, pelo conjunto total dos critérios, foi estabelecido que um valor igual ou superior a 0,70 para cada critério considerava-o válido ${ }^{15}$. Os dados referentes à caracterização dos participantes do estudo (especialistas e estudantes), bem como dos resultados da aplicação da EDS foram analisados pela estatística descritiva. A fidedignidade da EDS, nesta amostra, foi avaliada pelo alfa de Cronbach, valores superiores a 0,70 indicaram adequada fidedignidade $^{18}$.

Seguiram-se os preceitos éticos estabelecidos pela Resolução no 466/2012 do Conselho Nacional de Saúde, de modo que a proposta do estudo foi apreciada e aprovada pelo Comitê de Ética em Pesquisa (CAAE: 91085318.6.0000.5504) com número do parecer 2.847.470.

\section{RESULTADOS}

Para compor o painel para validação de conteúdo do cenário, foram convidados treze juízes, dos quais nove aceitaram participar do estudo. O painel foi composto por seis enfermeiros(as), uma obstetriz, um fisioterapeuta e uma médica. Sobre as áreas de experiência, quatro $(44,4 \%)$ apresentavam em simulação entre um a cinco anos; três (33,3\%) em cuidados paliativos entre oito e 12 anos e um $(11,1 \%)$ em comunicação durante cinco anos.

O IVC foi satisfatório para a análise geral do cenário pelos especialistas com valor de 0,84 , sendo que os valores para cada critério analisado variaram de 0,44 a 1 (Tabela 1).

O IVC dos elementos do cenário sobre os objetivos de aprendizagem, design, preparação dos participantes e instruções para a atriz foram inferiores a 0,70 , que foi o valor mínimo estabelecido previamente para serem válidos. Porém, os especialistas apontaram sugestões para adequação destes elementos, com vistas a melhorar a clareza da redação e o realismo do cenário, que foram integralmente incorporadas na versão final do cenário.

Portanto, foi sugerida ao objetivo geral de aprendizagem a alteração do verbo 'treinar' para 'desenvolver', 'empregar' ou 'demonstrar' e o acréscimo de descrição de quais estratégias de comunicação seriam utilizadas. Deste modo, o objetivo geral passou de "Treinar habilidades de comunicação de notícias difíceis, aplicando estratégias específicas de comunicação" para "Empregar a habilidade de comunicação de notícias difíceis, aplicando as estratégias SPIKES, NURSE ou Ask Tell Ask".

Sobre o design do cenário, os juízes apontaram sugestões para o preparo dos materiais, a fim de explicitar que os dispositivos intravenoso e de oxigenoterapia estejam conectados no manequim toddler, além de mudanças na hierarquização propostas pelos papéis dos participantes da simulação. Com isso, a proposta inicial era que dois participantes desempenhariam papeis de enfermeiro e estagiário, com a avaliação dos juízes os dois participantes desempenharam o mesmo papel, de enfermeiros.

Com base nas sugestões dos juízes, no elemento preparação dos participantes foi incluído o conteúdo da aula expositiva e dialogada sobre estratégias de comunicação de notícias difíceis, alterações na redação do termo "cuidados paliativos exclusivos" para "cuidados paliativos gerais", utilização de um nome fictício para a empresa citada no caso, informar que a mãe da criança é religiosa sem mencionar uma religião específica para não gerar conflito religioso, dar clareza no caso de que a criança é filho único e que a mãe sabe sobre a gravidade do quadro.

Nos elementos da progressão da simulação e das instruções para as atrizes, foram sugeridas inclusões acerca de maior detalhamento sobre os papeis das atrizes que participam da simulação e de um possível desfecho, caso o participante não consiguisse prosseguir na simulação por elevado envolvimento emocional, que foram prontamente incorporados.

Deste modo, foi completado um cenário intitulado Comunicação de Notícias Difíceis em contexto de Cuidados Paliativos, voltado aos estudantes de graduação em Enfermagem que estavam cursando ou cursaram disciplinas nas áreas de comunicação e saúde da criança, com propósito educacional de treinamento de habilidades de comunicação. O objetivo geral de aprendizagem foi de empregar a habilidade de comunicação de notícias difíceis, aplicando as estratégias SPIKES, NURSE ou Ask Tell Ask e os específicos foram: aplicar os passos do protocolo de SPIKES na condução de uma notícia difícil, aplicar as estratégias NURSE para responder às emoções geradas com a comunicação de uma notícia difícil e aplicar a estratégia Ask Tell Ask para explorar mais informações. A modalidade desta simulação foi cênica, com previsão de duração de 60 minutos (15 minutos destinados ao prebriefing e briefing, 15 minutos para a simulação e 30 minutos para o debriefing).

A preparação dos participantes ocorreu por meio de uma intervenção educativa denominada "Oficina para Comunicação de Notícias Difíceis", compreendendo aula expositiva e dialogada sobre notícias difíceis e estratégias de comunicação no contexto 
das notícias difíceis (SPIKES, NURSE, Ask Tell Ask), com duração de três horas.

Este cenário previa a participação de dois estudantes voluntários e duas atrizes com papeis de médica e mãe de uma criança internada para tratamento oncológico. $\mathrm{O}$ ambiente teve o realismo ajustado de modo que se assemelhasse a uma unidade de internação pediátrica, no leito havia um manequim toddler com dispositivo intravenoso, soro fisiológico de $1000 \mathrm{~mL}$ com identificação; equipo e fixação e cateter nasal instalados, estetoscópio pendurado em suporte de soro, frasco umidificador de oxigênio conectado ao fluxômetro, mesa de cabeceira com brinquedos, sacola com itens pessoais da criança, garrafa com água e copos descartáveis, relógio de parede marcando a hora do momento, três cadeiras, bloco para anotações e caneta.

O briefing apresentado foi "Vocês são enfermeiros(as) do setor de oncologia pediátrica e uma técnica de enfermagem, após aferir os sinais vitais da criança chamada João, pede para que a médica e vocês se direcionem ao quarto imediatamente. João, cinco anos, com diagnóstico médico de osteossarcoma em tíbia direita, fez tratamento com quimioterapia e radioterapia há um ano, evoluiu com metástase e encontra-se em cuidados paliativos gerais há 4 meses. A criança e família estavam em acompanhamento por equipe multiprofissional e internados há 30 dias. No momento estava dormindo com a mãe".

Na progressão da simulação, os participantes em companhia da atriz, que desempenha o papel de médica, identificam que a criança faleceu e precisam comunicar esta informação à mãe. A partir da previsão das cenas e possibilidades de desfechos deste cenário, era esperado que os estudantes desempenhassem as ações: apresentar-se, aproximar-se ou tocar a mãe, pegar cadeiras para sentar-se próximos à mãe, usar o silencio para que a mãe expresse sua emoção, transmitir atenção e calma, perguntar sobre o que a mãe estava entendendo acerca do quadro do João, aplicar NURSE, retomar a notícia e empregar linguagem adequada, direta, sem uso de termo e/ou jargões e ofertar ajuda (verificar se é de desejo da mãe que chamem alguém da família, receber apoio espiritual/religioso, ficar sozinha com a criança, participar dos cuidados com o corpo).

Três desfechos foram propostos a depender do desempenho dos participantes, primeiro: caso ofertassem ajuda prontamente e identificassem os desejos da mãe a simulação era encerrada; segundo: na ausência da oferta de ajuda a atriz que representava a mãe perguntaria o que ela deveria fazer naquele momento (pista) e uma vez que os estudantes oferecessem apoio e identificassem os desejos da mãe, a simulação era encerrada e, terceiro: diante de grande envolvimento emocional ou não disponibilização de ajuda à mãe, mesmo com a pista oferecida anteriormente, a médica retorna à simulação e os auxilia na identificação de ajuda e desejos da mãe, com encerramento do cenário. Após a simulação, os participantes foram conduzidos para o debriefing que foi pautado no modelo estruturado.

O teste do cenário ocorreu com 15 estudantes de graduação enfermagem, sendo $10(66,6 \%)$ matriculados no quarto semestre, dois $(13,3 \%)$ no sexto semestre, dois $(13,3 \%)$ no segundo semestre e um $(6,8 \%)$ no primeiro semestre. A idade variou entre 17 e 33 anos, e predominou a participação de estudantes do gênero feminino ( $n=14 ; 93,3)$.

Quando questionados sobre a vivência de situações que exigiam a comunicação de notícia difícil em campo de estágio, todos relataram não ter presenciado, 14 (93,3\%) relataram que o tema não foi trabalhado durante o curso e $12(86,6 \%)$ nunca tinham participado de atividades extracurriculares com esta finalidade.

$\mathrm{Na}$ Tabela 2, estão os resultados da avaliação do design do cenário pelos estudantes, a partir das respostas na EDS, bem como a análise da confiabilidade desta escala nesta amostra. Destaca-se que os resultados do alfa de Cronbach evidenciam adequada fidedignidade dos dados a partir da aplicação da EDS, com valores superiores a 0,70 , com exceção dos fatores Resolução de problemas e Realismo.

Os estudantes, a partir das respostas nas subescalas da EDS, consideraram o cenário adequado, uma vez que todos ( $n=15 ; 100 \%)$ concordaram com a adequação dos "Objetivos e Informações", "Apoio", "Feedback/Reflexão" e "Realismo", pois responderam os itens 4 e 5 (Concordo e Concordo fortemente com a afirmação) para cada item destes fatores, indicando atendimento destes elementos na simulação. O fator "Resolução de Problemas" obteve concordância de 93,3\%.

\section{DISCUSSÃO}

No contexto de assistência à criança em cuidados paliativos, questões sobre dificuldade dos profissionais em compartilhar

Tabela 2. Descrição da média e confiabilidade da Escala de Design da Simulação. São Carlos, SP, Brasil, 2018. (n=15).

\begin{tabular}{lcc}
\multicolumn{1}{c}{ Escala de Design da Simulação } & Média & alfa de Cronbach \\
\hline Objetivos e Informações & 4,78 & 0,8 \\
Apoio & 4,9 & 0,9 \\
Resolução de Problemas & 4,88 & 0,6 \\
Feedback/Reflexão & 4,88 & 0,88 \\
Realismo & 4,83 & 0,45 \\
Total & 4,85 & 0,89 \\
\hline
\end{tabular}

Fonte: Dados de pesquisa. 
notícias difíceis aos pais são evidentes e estão relacionadas à forma como os pais percebem a qualidade do cuidado ofertado ${ }^{19,20}$ Além disso, a comunicação e apoio recebidos no final de vida vivenciados pelos pais têm impacto importante na experiência e no enfrentamento do luto ${ }^{21}$.

Estas dificuldades são frequentes na descrição de pais que vivenciaram os cuidados paliativos com seus filhos, evidenciadas em estudos na área ${ }^{19,21}$. Na Índia, pais que perderam suas crianças com câncer, relataram que não receberam apoio emocional no momento que foram informados sobre a morte de seus filhos, além disso, esta comunicação foi de forma breve, insensível e não empática ${ }^{21}$. Pais holandeses de crianças que estiveram em cuidados paliativos relataram desejo de ter mais tempo para conversar com os profissionais da saúde, de receber informações honestas e realistas em ambiente e momento adequados, de terem suas dúvidas esclarecidas, além de receber ajuda para tomar decisões ${ }^{19}$.

Embora a comunicação seja um componente transcendental do cuidado em saúde, a comunicação de notícias difíceis é frequentemente considerada uma situação desafiadora, capaz de gerar sofrimento emocional em parte de profissionais da área da saúde, que pode ser abrandado pelo fortalecimento das habilidades de comunicação21,22. Diante disso, a simulação aparece como uma estratégia potente e frequentemente utilizada para desenvolvimento e aperfeiçoamento das habilidades de comunicação em cuidados paliativos, em especial para as notícias difíceis ${ }^{10}$.

Revisões da literatura reforçaram o benefício da simulação para fortalecimento das habilidades de comunicação de notícias difíceis em cuidados paliativos, mas ponderam a importância de se construir cenários que consideram a interprofissionalidade, que possuam objetivos de aprendizagem claros e que sejam validados ${ }^{7,9}$.

No processo de elaboração do cenário para comunicação de notícias difíceis, a interprofissionalidade foi levada em consideração, ao incluir os papeis de médico e enfermeiro, pois se entende que a emissão de notícias difíceis ocorre de maneira completa quando realizada em equipe e que os enfermeiros lidam, principalmente, com emoções geradas por estas informações ${ }^{14}$.

No contexto de assistência à criança, há indicação de que a comunicação de notícias difíceis seja feita pelo profissional que acompanha a criança, e quando for de teor técnico, indica-se que seja feita pelo médico, porém, com a presença de outros profissionais envolvidos no cuidado à criança e familiares, pois cada profissão possui atribuição e competências específicas, que somadas compõem uma abordagem completa de atenção à saúde ${ }^{23}$

De maneira específica, o/a enfermeiro/a tem distintos papeis no momento da comunicação de notícias difíceis, seja como responsável por transmitir a notícia, acompanhar outro profissional neste momento e manejar junto aos pacientes e/ ou familiares o impacto promovido pela notícia ${ }^{3,23}$. Ressalta-se que é possível que em grande parte das vezes, o papel do/a enfermeiro/a envolve falar com pessoas que já receberam uma notícia difícil, e por isso, as habilidades de comunicação serão necessárias para acessar questões voltadas à dimensão espiritual, esclarecer dúvidas, auxiliar na tomada de decisão e em resoluções necessárias para o contexto de saúde, oferecer apoio, manejar emoções, sentimentos e medos ${ }^{24}$.

Entende-se que a emoção seja um fator que favorece o aprendizado com a simulação, no entanto, se compreende a importância do equilíbrio entre emoção e integridade do participante, em que pese este ser um quesito de boas práticas para uso da simulação ${ }^{13,25,26}$. Ao ferir a integridade do participante durante a simulação, podem ocorrer comportamentos imprevistos e/ou interferência nos resultados, dificuldade dos participantes em imergirem na experiência, perda do ambiente de aprendizado seguro, além de consequências mais sérias como comprometimento da carreira e autoestima ${ }^{27}$.

A eficácia da simulação está completamente relacionada ao rigor de planejamento no cenário, especialmente à garantia do realismo do ambiente, materiais, manequins, atores, recursos, caso clínico, ponto de partida da simulação e progressão das ações durante a atividade ${ }^{12,27,28}$. Todos estes aspectos convergem para atingir a fidelidade da atividade simulada, ou seja, a criação de um contexto capaz de reproduzir ao participante a percepção de estar em um ambiente real e promover a aprendizagem ${ }^{11}$.

A validação de conteúdo dos cenários permite analisar o quanto todos os elementos incorporados correspondem ao conteúdo, ou seja, quão bem foi reunido o material sobre o conteúdo ${ }^{15}$. Assim, o teste dos cenários em elaboração faz sentido, pois permite receber análise e avaliação de elementos próprios das atividades simuladas pelos participantes, em uma dimensão distinta da validação de conteúdo, ou seja, do desenvolvimento da simulação.

O teste do cenário com o público alvo é uma recomendação de boas práticas do planejamento de cenários pelo INACSL, considerando que seja uma forma de garantir que a simulação seja eficaz e ocorra conforme esperado pelos elaboradores, com vistas a identificar confusão ou ausência de elementos que possam ser corrigidas a tempo ${ }^{13}$.

A utilização de um instrumento de avaliação dos cenários pelos participantes é também uma diretriz de boas práticas no planejamento de cenários, fortalece a validação, consistência e confiabilidade do cenário ${ }^{11}$. Desde a validação da EDS para utilização no Brasil ${ }^{17}$, vários estudos a utilizaram, como forma de analisar a estruturação dos cenários ${ }^{14,28-30}$, no entanto, o presente estudo ampliou as investigações sobre a fidedignidade desta medida, e analisou o alfa de Cronbach, que foi adequado para o escore total e a maioria dos fatores ${ }^{18}$.

Deste modo, apreende-se que os resultados obtidos sobre a fidedignidade da EDS nesta amostra, são confiáveis e precisos, porém a amostra com um número pequeno de participantes pode ter influenciado os valores de alfa de Cronbach obtidos nos fatores "Resolução de Problemas" e "Realismo", que foram menores que 0,70 e inferiores ao limite estabelecido como adequado ${ }^{18}$.

Por fim, é importante que os profissionais reconheçam que no contexto do cuidado à criança há grande valorização por parte 
dos pais e das próprias crianças quanto à comunicação com os profissionais de saúde. Os pais esperam que a comunicação se estabeleça de forma clara, honesta, objetiva e empática, além disso, associam boas habilidades de comunicação com a confiança no profissional ${ }^{22}$.

Ainda, é importante reforçar a singularidade da comunicação de notícias difíceis no contexto pediátrico de cuidados paliativos, pois sempre vai além da pessoa que precisa de cuidados, envolvendo também seus pais ou responsáveis. Além disso, há de considerar a utilização de estratégias de comunicação que atendam os aspectos intelectuais, culturais e psicológicos da criança e sua família ${ }^{4,23}$.

Portanto, a formação para ampliar as habilidades de comunicação de notícias difíceis se mostra fundamental para qualificação da formação dos profissionais de saúde, bem como do cuidado prestado.

\section{CONCLUSÃO E IMPLICAÇÕES PARA A PRÁTICA}

Um cenário para comunicação de notícias difíceis foi rigorosamente elaborado, adotando diretrizes de entidades especializadas em simulação, bem como em evidências científicas sobre o tema, seguido de validação de conteúdo por um painel de juízes que julgaram os elementos apresentados no cenário adequados, claros, capazes de promover os objetivos propostos e, portanto, válidos em relação ao seu conteúdo. Por fim, o teste do cenário junto à população alvo reafirmou sua adequação pelos participantes. Recomenda-se a realização de estudos futuros com vistas a avaliar a eficácia deste cenário na melhora da habilidade de estudantes para comunicação de notícias difíceis.

Como limitação deste estudo, aponta-se que o cenário, após a análise dos especialistas e as alterações realizadas a partir do que foi proposto por este painel, não passou por uma nova rodada para validação de seu conteúdo, a fim de se certificar que o IVC estaria em conformidade com o esperado previamente (IVC $\leq 0,70)$.

\section{CONTRIBUIÇÕES DOS AUTORES}

Desenho do estudo. Daiane de Assis Flausino. Andressa Rueda de Oliveira. Maira Deguer Misko. Aline Helena Appoloni Eduardo.

Aquisição de dados. Daiane de Assis Flausino. Andressa Rueda de Oliveira. Maira Deguer Misko. Aline Helena Appoloni Eduardo.

Análise de dados e interpretação dos resultados. Daiane de Assis Flausino. Andressa Rueda de Oliveira. Maira Deguer Misko. Aline Helena Appoloni Eduardo.

Redação e revisão crítica do manuscrito. Daiane de Assis Flausino. Andressa Rueda de Oliveira. Maira Deguer Misko. Aline Helena Appoloni Eduardo.

Aprovação da versão final do artigo. Daiane de Assis Flausino. Andressa Rueda de Oliveira. Maira Deguer Misko. Aline Helena Appoloni Eduardo.
Responsabilidade por todos os aspectos do conteúdo e a integridade do artigo publicado. Daiane de Assis Flausino. Andressa Rueda de Oliveira. Maira Deguer Misko. Aline Helena Appoloni Eduardo.

\section{EDITOR ASSOCIADO}

Rafael Celestino da Silva (1)

\section{EDITOR CIENTÍFICO}

Marcelle Miranda da Silva (D)

\section{REFERÊNCIAS}

1. Bellaguarda MLR, Knihs NS, Canever BP, Tholl AD, Alvarez AG, Teixeira GC. Realistic simulation as a teaching tool in critical situation communication in palliative care. Esc Anna Nery. 2020;24(3):e20190271. http://dx.doi.org/10.1590/2177-9465-ean-2019-0271.

2. Fontes CMB, Menezes DV, Borgato MH, Luiz MR. Communicating bad news: na integrative review of the nursing literature. Rev Bras Enferm. 2017;70(5):1089-95. http://dx.doi.org/10.1590/0034-7167-2016-0143. PMid:28977239.

3. Warnock C, Buchanan J, Tod AM. The difficulties experience by nurses and healtcare staff involved in the process of breaking bad news. $J$ Adv Nurs. 2017;73(7):1632-45. http://dx.doi.org/10.1111/jan.13252. PMid:28072478.

4. Buckman RA. Breaking bad news: why is it still so difficult? Br Med J 1984;288(6430):1597-9. http://dx.doi.org/10.1136/bmj.288.6430.1597. PMid:6426658.

5. Yakhforoshha A, Emami SAH, Shahi F, Shahsavari S, Cheraghi M Mojtahedzadeh R et al. Effectiveness of integrating simulation with artbased teaching strategies on oncology fellows'performance regarding breaking bad news. J Cancer Educ. 2019;34(3):463-71. http://dx.doi. org/10.1007/s13187-018-1324-x. PMid:29468497.

6. Bumb M, Keefe J, Miller L, Overcash J. Breaking bad news: an evidencebased review of communication models for oncology nurses. Clin J Onco Nurs. 2017;21(5):573-80. http://dx.doi.org/10.1188/17.CJON.573-580 PMid:28945712.

7. Smith MB, Macieira TGR, Bumbach MD, Garbutt SJ, Citty SW, Stephen A et al. The use of simulation to teach nursing students and clinicians palliative care and end-of-life communication: a systematic review. Am J Hosp Palliat Care. 2018;35(8):1140-54. http://dx.doi. org/10.1177/1049909118761386. PMid:29514480.

8. Brown CE, Back AL, Ford DW, Kross EK, Downey L, Shannon SE et al Self-assessment scores improve after simulation-based palliative care communication skill workshops. Am J Hosp Palliat Care. 2018;35(1):4551. http://dx.doi.org/10.1177/1049909116681972.

9. Oliveira RRC, Medeiros SM, Vitor AF, Lira ALBC, Martins JCA, Araújo MS. Types and purposes of the simulation in undergraduate nursing education: integrative literature review. Rev Baiana Enferm. 2016 jul/ set;30(3):1-11. http://dx.doi.org/10.18471/rbe.v30i3.16589.

10. Kozhevnikov D, Morrison LJ, Ellman MS. Simulation training in palliative care: state of the art and future directions. Adv Med Educ Pract. 2018;9:915-24. http://dx.doi.org/10.2147/AMEP.S153630.

11. Lioce L, Lopreiato J, Downing D, Chang TP, Robertson JM, Anderson $M$, et al. Healtcare simulation dictionary. 2nd ed. Rockville: Agency for Healtcare Research and Quality; 2020. http://dx.doi.org/10.23970/ simulationv2.

12. Kaneko RMU, Lopes MHBM. Realistic health care simulation scenario: what is relevant for its design? Rev Esc Enferm USP. 2019;53:e03453. http://dx.doi.org/10.1590/s1980-220x2018015703453. PMid:31166535.

13. International Nursing Association for Clinical Simulation and Lerning, INACSL Standards Committee. INASCL standards of best pratice: simulationSM simulation design. Clin Simul Nurs. 2016;12:S5-12. http:// dx.doi.org/10.1016/j.ecns.2016.09.005. 
14. Dias AAL. A simulação clínica como estratégia de ensino de medidas de prevenção e controle de infecções relacionadas à assistência à saúde [tese]. São Carlos: Programa de Pós-graduação em Enfermagem, Universidade Federal de São Carlos;2019 [citado 8 mar 2021]. Disponível em: https://repositorio.ufscar.br/handle/ufscar/12110

15. Rubio DM, Berg-Weger M, Tebb SS, Lee ES, Rauch S. Objectifying content validity: conducting a content validity study in social work research. Soc Work Res. 2003;27(2):94-105. http://dx.doi.org/10.1093/ swr/27.2.94.

16. Eduardo AHA, Mendes AA, Binotto CCS, Tognoli SH, Tucci AMGB Scenario for a simulation of health services' waste: a methodological study. Online Braz J Nurs. 2016;15(4):611-6. http://dx.doi.org/10.17665/16764285.20165672.

17. Almeida RS, Mazzo A, Martins JCA, Pedersoli CE, Fumincelli L, Mendes IAC. Validação para a língua portuguesa da Simulation Design Scale. Texto Contexto Enferm. 2015;24(4):934-40. http://dx.doi.org/10.1590/01040707201500004570014

18. Terwee CB, Bot SDM, de Boer MR, van der Windt DAWM, Knol DL, Dekker J et al. Quality criteria for clinimetric studies of health status questionnaires. J Clin Epidemiol. 2007;61(1):34-42. http://dx.doi. org/10.1016/j.jclinepi.2006.03.012. PMid:17161752.

19. Brouwers M, van Weel C, Laan R, van Weel-Baumgarten E. Training undergraduates skills in breaking bad news: how students value educators' feedback. J Cancer Educ. 2019 dez;34(6):1103-6. http:// dx.doi.org/10.1007/s13187-018-1415-8. PMid:30128954.

20. Brock KE, Tracewski M, Allen KE, Klick J, Petrillo T, Hebbar KB. Simulation-based palliative care communication for pediatric critical care fellows. Am J Hosp Palliat Care. 2019;36(9):820-30. http://dx.doi. org/10.1177/1049909119839983. PMid:30974949.

21. Das MK, Arora NK, Chellani HK, Debata PK, Meena KR, Rasaily R et al Perceptions of the parents of deceased children and of healthcare providers about end-of-life communication and breaking bad news at a tertiary care public hospital in India: a qualitative exploratory study. PLoS One. 2021;16(3):e0248661. http://dx.doi.org/10.1371/journal. pone.0248661. PMid:33735296.

22. González-Cabrera M, Ortega-Martínez AR, Martínez-Galiano JM, Hernández-Martínez A, Parra-Anguita L, Frías-Osuna A. Design and validation of a questionnaire on communicating bad news in nursing a pilot study. Int J Environ Res Public Health. 2020;17(2):457. http:// dx.doi.org/10.3390/ijerph17020457. PMid:31936751.

23. Zanon BP, Cremonese L, Ribeiro AC, Padoin SMM, Paula CC Communication of bad news in pediatrics: integrative review. Rev Bras Enferm. 2020;73(Supl.4):e20190059. http://dx.doi.org/10.1590/00347167-2019-0059. PMid:32785469.

24. Wittenberg E, Reb A, Kanter E. Communicating with patients and families around difficult topics in cancer care using the COMFORT communication curriculum. Semin Oncol Nurs. 2018;34(3):264-73 http://dx.doi.org/10.1016/j.soncn.2018.06.007. PMid:30100368.

25. International Nursing Association for Clinical Simulation and Lerning INACSL Standards Committee. INACSL standards of best practice: simulationSM Professional integrity. Clin Simul Nurs. 2016;12:S30-3 http://dx.doi.org/10.1016/j.ecns.2016.09.010.

26. Benishek LE, Lazzara EH, Gaught WL, Arcaro LL, OkudaY, Salas E. The Template of Events for Applied and Critical Healthcare Simulation (TEACH Sim): a tool for systematic simulation scenario design. Simul Healthc 2015;10(1):21-30. http://dx.doi.org/10.1097/SIH.0000000000000058.

27. Leon CGRM, Silva AK, Ribeiro LM, Brasil CC, Guarda LEA, Fonseca LMM. Construção e validação de casos clínicos para utilização no ensino de enfermagem no contexto materno-infantil. Rev Enf Ref. 2018 set;IV(18):51-62. http://dx.doi.org/10.12707/RIV18013.

28. Silva JLG, Oliveira-Kumakura ARS. Simulação clínica para ensino da assistência ao paciente com ferida. Rev Bras Enferm. 2018;71(Supl. 4):1785-90. http://dx.doi.org/10.1590/0034-7167-2017-0170 PMid:30088654.

29. Negri EC, Pereira GAJ, Cotta CK Fo, Franzon JC, Mazzo A. Construção e validação de cenário simulado para assistência de enfermagem a pacientes com colostomia. Texto Contexto Enferm. 2019;28:e20180199. http://dx.doi.org/10.1590/1980-265x-tce-2018-0199.

30. Bergamasco EC, Murakami BM, Cruz DALM. Uso da Escala de Satisfação dos Estudantes e Autoconfiança com a Aprendizagem (ESEAA) e da Escala do Design da Simulação (EDS) no ensino de enfermagem: relato de experiência. Sci Med. 2018;28(3):ID31036. http://dx.doi. org/10.15448/1980-6108.2018.3.31036. 